\title{
checkCIF/PLATON report
}

No syntax errors found. CIF dictionary Interpreting this report

\section{Datablock: subTAPOH}

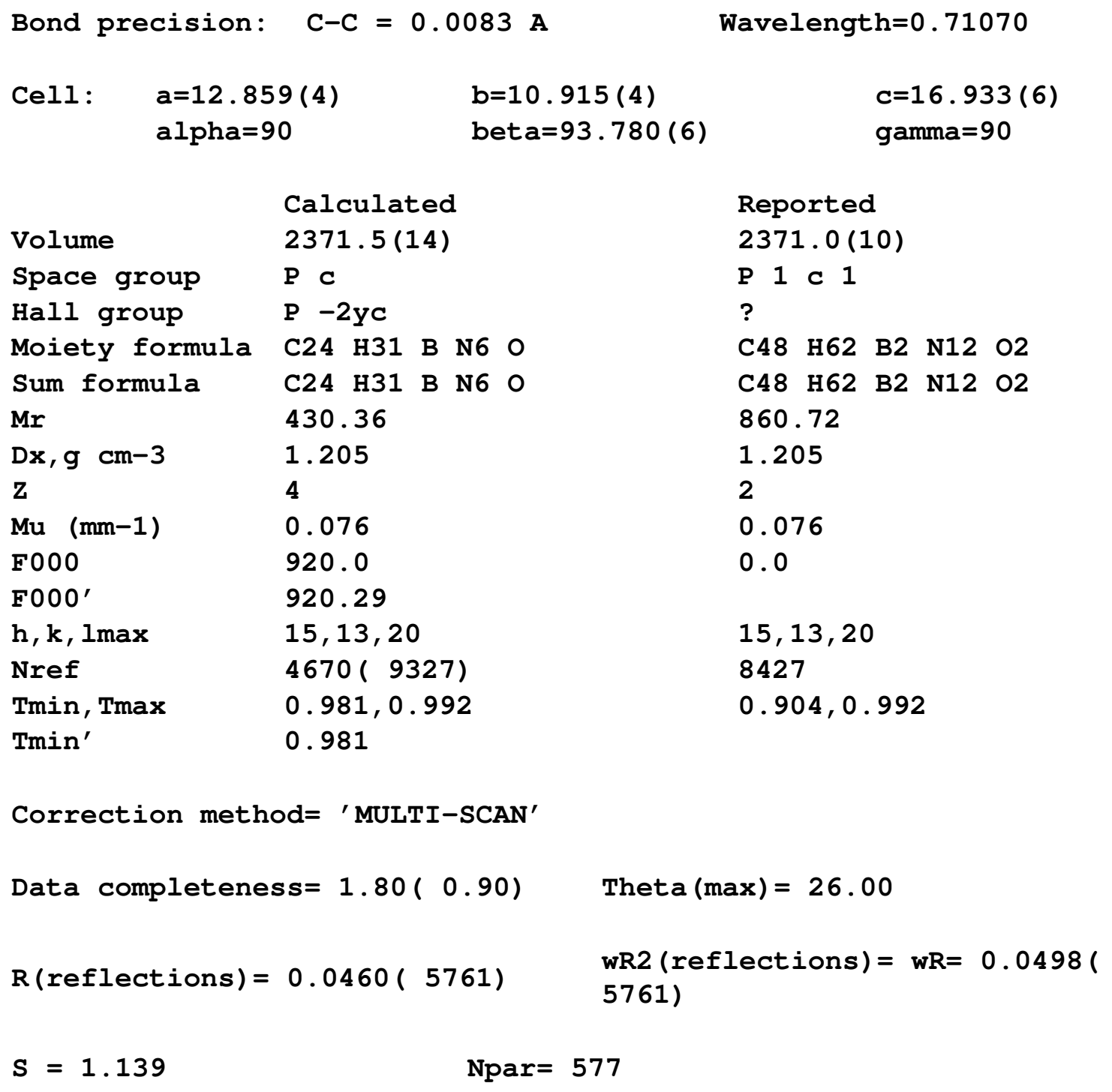

Alert level $A$

WEIGHO1_ALERT_1_A Unit weights are not acceptable for submissions to Acta Crystallographica section $\mathrm{C}$. n.b. unit is however a legal CIF keyword. 


\begin{tabular}{|l|}
\hline PLAT 390_ALERT_3_A \\
\hline PLAT 390_ALERT_3_A \\
\hline PLAT 3 91_ALERT_3_A \\
\hline PLAT 391_ALERT_3_A \\
\hline PLAT3 91_ALERT_3_A \\
\hline
\end{tabular}

Deviating Methyl C44

Deviating Methyl C46

Deviating Methyl C24

Deviating Methyl C24

Deviating Methyl C44
$\mathrm{X}-\mathrm{C}-\mathrm{H}$ Bond Angle .....

$\mathrm{X}-\mathrm{C}-\mathrm{H}$ Bond Angle .....

$\mathrm{H}-\mathrm{C}-\mathrm{H}$ Bond Angle .....

$\mathrm{H}-\mathrm{C}-\mathrm{H}$ Bond Angle .....

$\mathrm{H}-\mathrm{C}-\mathrm{H}$ Bond Angle .....
126.59 Deg. $134.37 \mathrm{Deg}$. 124.31 Deg. 77.53 Deg. 85.49 Deg.

\section{Alert level B}

PLAT024_ALERT_4_B Merging of Friedel pairs is STRONGLY indicated. PLAT220_ALERT_2_B Large Non-Solvent C Ueq (max)/Ueq(min) ... PLAT351_ALERT_3_B Long C-H Bond (0.96A) C44 - $\mathrm{H} 49$... PLAT390_ALERT_3_B Deviating Methyl C18 X-C-H Bond Angle ...... PLAT390_ALERT_3_B Deviating Methyl C38 X-C-H Bond Angle ...... PLAT390_ALERT_3_B Deviating Methyl C40 PLAT413_ALERT_2_B Short Inter XH3 .. XHn $\mathrm{X}-\mathrm{C}-\mathrm{H}$ Bond Angle ..... H2O $\mathrm{H}$ H47 ..

$$
\begin{aligned}
& 4.21 \text { Ratio } \\
& 1.20 \text { Ang. } \\
& 95.63 \text { Deg. } \\
& 96.67 \text { Deg. } \\
& 122.19 \text { Deg. } \\
& 1.98 \text { Ang. }
\end{aligned}
$$

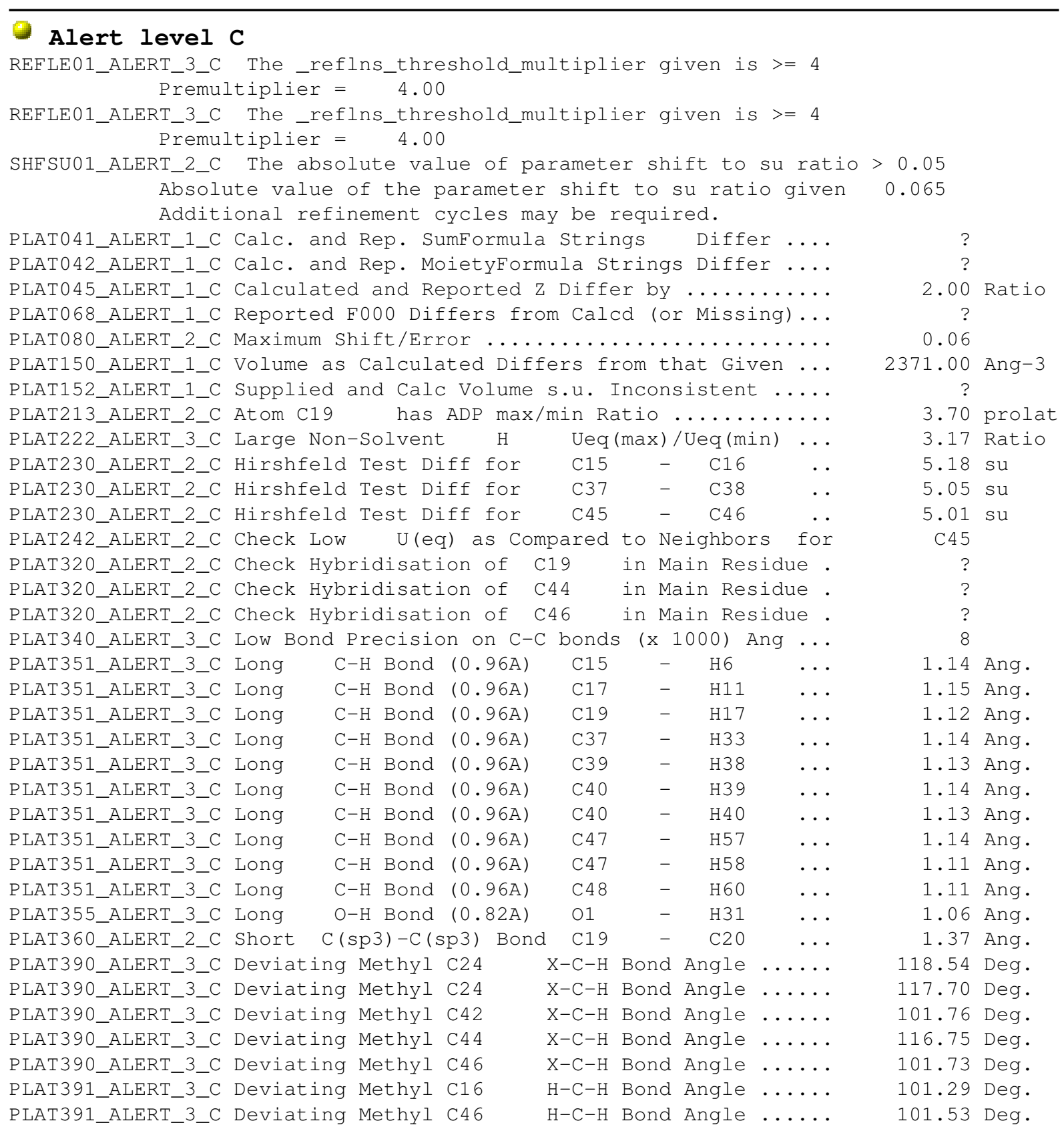




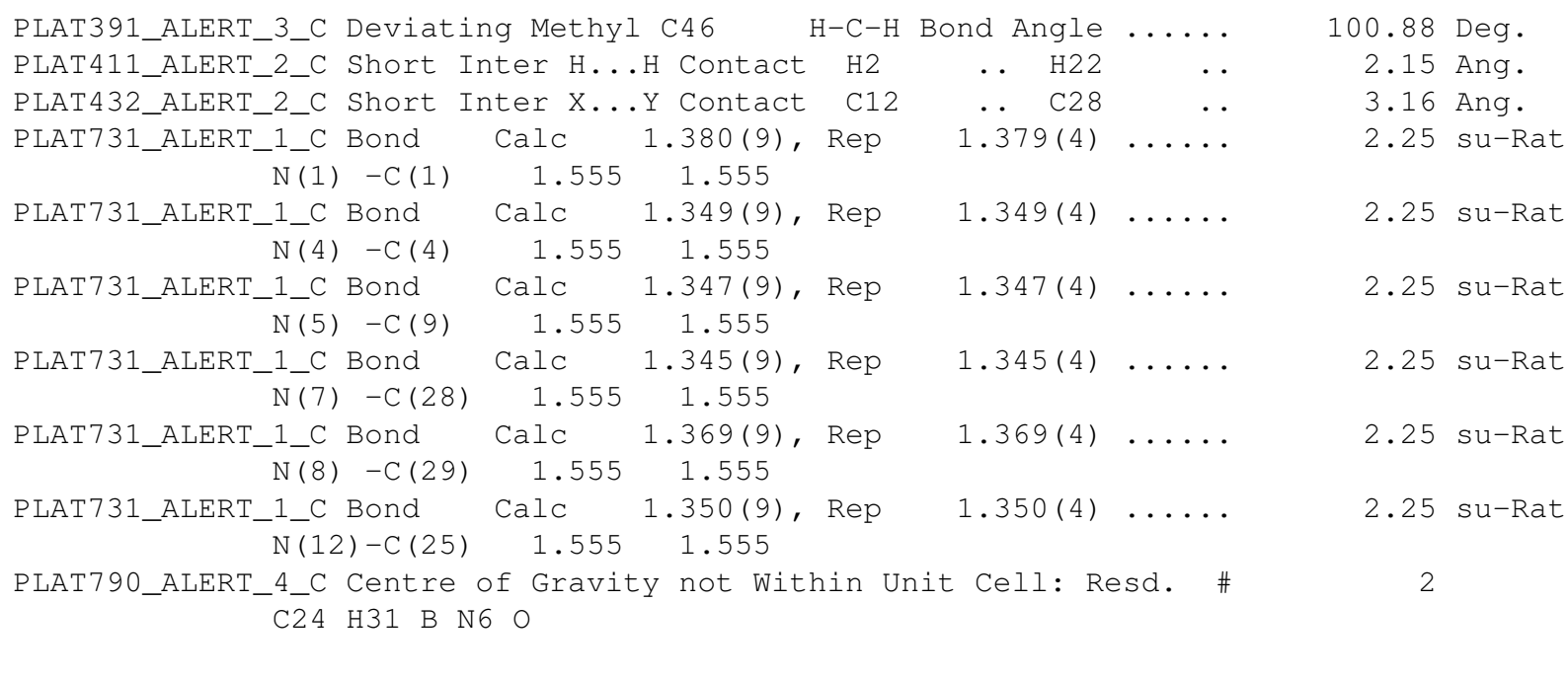

Alert level G

REFLT03_ALERT_4_G ALERT: MoKa measured Friedel data cannot be used to determine absolute structure in a light-atom study EXCEPT under VERY special conditions. It is preferred that Friedel data is merged in such cases.

From the CIF:_diffrn_reflns_theta_max 26.00

From the CIF:_reflns_number_total 8427

Count of symmetry unique reflns 4670

Completeness (_total/calc) 180.45\%

TEST3: Check Friedels for noncentro structure

Estimate of Friedel pairs measured 3757

Fraction of Friedel pairs measured 0.804

Are heavy atom types $\mathrm{Z}>\mathrm{Si}$ present no

9 ALERT level $\mathbf{A}=$ In general: serious problem

7 ALERT level $\mathbf{B}=$ Potentially serious problem

49 ALERT level $\mathbf{C}=$ Check and explain

1 ALERT level $\mathbf{G}=$ General alerts; check

13 ALERT type 1 CIF construction/syntax error, inconsistent or missing data

16 ALERT type 2 Indicator that the structure model may be wrong or deficient

34 ALERT type 3 Indicator that the structure quality may be low

3 ALERT type 4 Improvement, methodology, query or suggestion 
A basic structural check has been run on your CIF. These basic checks will be run on all CIFs submitted for publication in IUCr journals (Acta Crystallographica, Journal of Applied Crystallography, Journal of Synchrotron Radiation); however, if you intend to submit to Acta Crystallographica Section $C$ or $E$, you should make sure that full publication checks are run on the final version of your CIF prior to submission.

Publication of your CIF in other journals

Please refer to the Notes for Authors of the relevant journal for any special instructions relating to CIF submission.

PLATON version of 12/07/2004; check.def file version of 12/07/2004

\section{Datablock subTAPOH - ellipsoid plot}

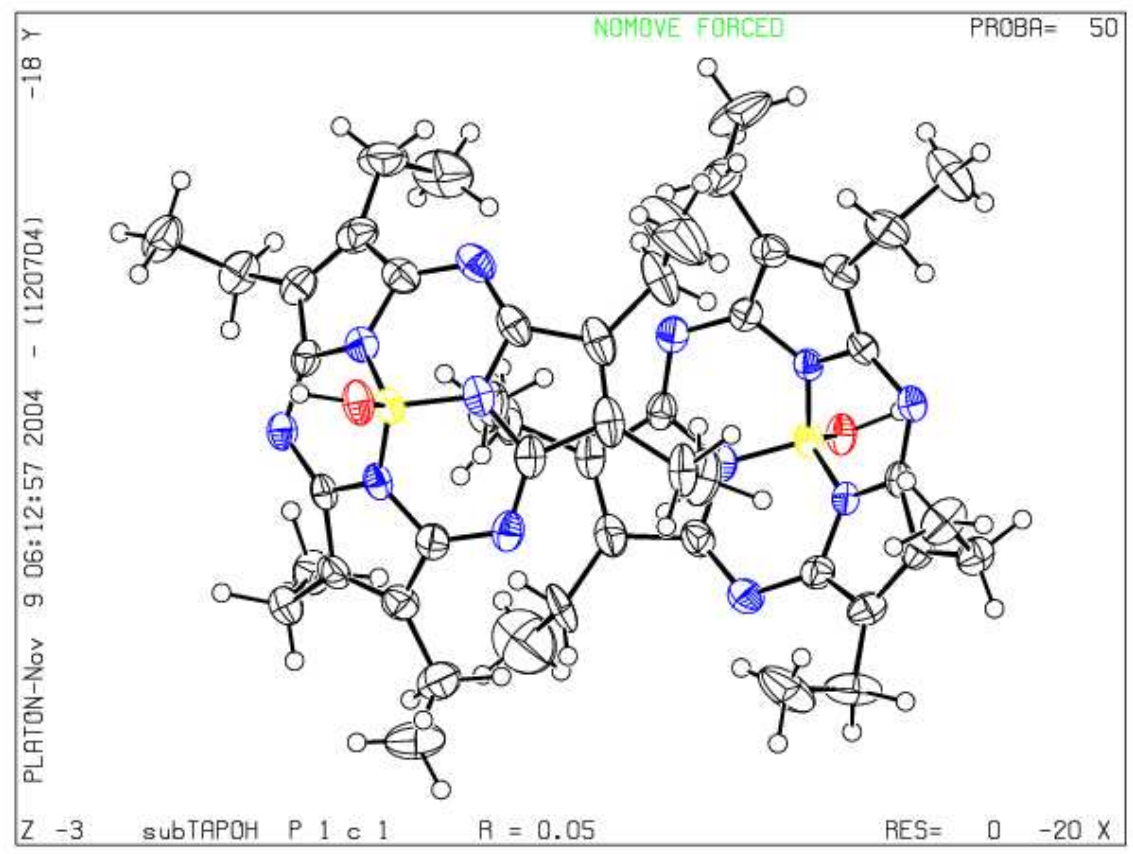

